\title{
Exploratory Analysis of Sony AIBO Users
}

\author{
Csaba Kertész and Markku Turunen ${ }^{1}$
}

\begin{abstract}
It is important to understand how the cultural background, the age and the gender influence the expectations towards social robots. Although past works studied the user adaptation for some months, the users with multiple years of ownership (heavy users) were not subjects of any experiment to compare these criteria over the years. This exploratory research examines the owners of the discontinued Sony AIBO because these robots have not been abandoned by some enthusiastic users and they are still resold on the secondhand market. 78 Sony AIBO owners were recruited on-line and their quantitative data were analyzed by four independent variables (age, gender, culture, length of ownership), user contribution and model preference points of view. The results revealed the motives to own these robots for years and how the heavy users perceived their social robots after a long period in the robot acceptance phase.
\end{abstract}

Keywords Quantitative research, heavy users, social robot, Sony AIBO.

\section{Introduction}

Nowadays more and more social robots are introduced onto the market and the user expectations must be understood for the researchers to execute successful long-term experiments and for the companies to create sustainable business plans. Graaf et al [7] developed a theoretical foundation to describe the relationship between the owner and its robot over time. Before the purchase, the consumer learns about the technology and makes the decision to acquire the product (pre-adoption phase). In the adoption and adaptation phases, the first experiences are gained with the robot at home. When the novelty effect fades away and the user expectations are met, daily routines are developed with the robot (incorporation phase). After six months, the owner gets emotionally attached to the robot as a personal object (identification phase), the robot is finally accepted for long-term use and the owner becomes a heavy user.

\footnotetext{
1 C. Kertész and M. Turunen

Faculty of Communications, University of Tampere, Tampere, Finland. E-mail: csaba.kertesz@ieee.org, markku.turunen@sis.uta.fi
}

This is a postprint of an article published in AI \& Society (Springer) https://doi.org/10.1007/s00146/ 018/ 0818/ 8
The commercial Sony AIBO robots still have a reachable, significant user base long after the discontinuation. Although these users can be analyzed from many perspectives, this study was based on a questionnaire to measure the perception of the robot and the technical expectations. The authors concentrated on these aspects to identify the key needs of this community because the literature examined the phases before the robot acceptance, but we do not have a good understanding of the heavy users beyond the identification phase. This paper is a preliminary step to build this knowledge.

\subsection{Robotics Questionnaires in the Literature}

This subsection reviews past works whose subjects filled robotics questionnaires and they were analyzed by independent variables (culture, age or gender). These participants were not robot owners and they were recruited from the internet, at universities or exhibitions thus their preferences represented the general public to some extent.

Zhan et al [24] studied the attitudes towards smart devices with mainly Australian participants to develop a Recommender System for particular tasks. The male participants were more likely to accept robots than the female and the age did not influence their ratings except the robotic pet which was disliked over 50 years of age.

Nomura et al [15] examined the visitors at an exhibition of interactive robots in Japan. Their questionnaire revealed that younger people liked the robots less than elder people and there was no difference in the behavior towards the robots from gender point of view. The visitors met with the robots once, therefore, these results were obtained in the pre-adoption phase.

Haring et al [18] developed a questionnaire to compare the European culture with Japanese regarding the emotions towards robots. It was found that Japanese people had higher exposure to the robots through the media, but they had less personal experiences than Europeans. This research did not found more positive attitude in the Japanese culture towards robots and Europeans accepted a human-like robot less than the Japanese people who saw the robots more as a machine.

Ezer [25] explored in his $\mathrm{PhD}$ thesis what kind of roles the American public expects from a robot. His questionnaire was sent via mail to random individuals in Atlanta (USA) and their answers indicated age-related differences in the desired robot tasks which can be considered for robot design to achieve better utility value for different age groups. However, the survey did not show 
any pictures of actual robots to the participants and these results were limited by the general imagination about the robots that the media and the television programs show us.

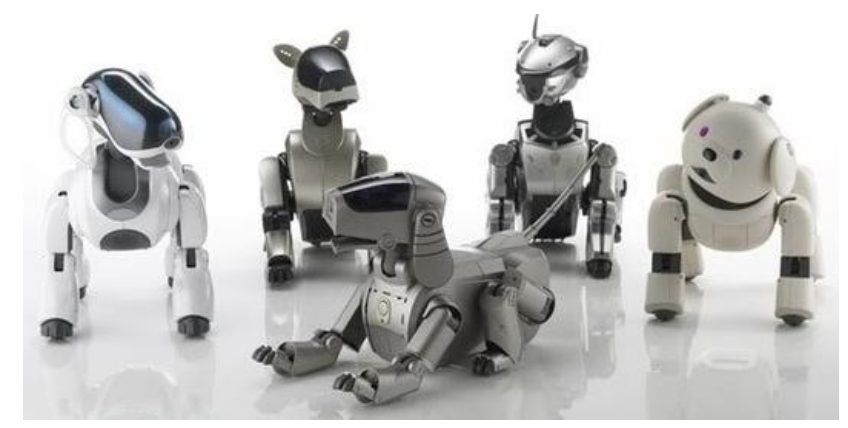

Fig. 1 Sony AIBO robots.

Bartneck et al [1] studied members of on-line robotics communities and university students from seven countries. Their Negative Attitude towards Robots Scale (NARS) questionnaire was analyzed with three independent variables (community, gender, culture) and the female participants were more positive about the social influence of the robots than males. The cultural background had a significant influence on the initial attitude towards robots although Japanese people were not so positive as stereotypically assumed. Interacting with AIBO had a positive effect on the results, but owning a robot did not improve the acceptance.

A humanoid robot (Robi) was exposed to Japanese and Australian people in [19] to find changes in the perception during an interaction. The first questionnaire filling before the session (Phase 0) reflected the cultural background and the initial expectations. The responses showed that the Australian participants liked the robot more and they rated its intelligence level higher than the Japanese people.

Nomura et al [20] measured the social acceptance towards humanoid robots in the Japanese population. They conducted a survey online with 1000 randomly-selected persons to represent all age groups between 20-60 years. The older generations had more positive expectations what could be further improved with human-robot interactions (HRI) or robotics news in the media. After HRI experiences, the younger generations had increased apprehension towards robots and their anxiety did not pass.

None of the studies in this subsection reviewed actual users of a robot or the effects after extensive interactions thus all participants were before pre-adoption stage. If the questionnaire in this paper can reveal similar tendencies to $[1,15,18,19,20,24]$ then the new results may generalize to the population.

\subsection{Studies in Human-Robot Interaction}

The literature of human-robot interaction (HRI) studied several robots in the past. A general observation was that the users have decreasing interest in the robots after their novelty fades away [6, 13]. To avoid losing attraction, social or other engaging capabilities must be identified to create robots for longer use. The long-term interaction was studied by Leite et al [11] in a comprehensive survey of exploratory papers of health care, education, work and home settings. They admitted that the reviewed experiments were carried out with limited number of users and the purpose of the longer duration was to let the participants to get comfortable with the experimental conditions. Their results suggested that people were happy to interact with the social robots for longer periods, but they proposed further analysis to confirm this hypothesis.

Graaf et al [7] investigated the social robot acceptance in domestic environment. 70 Karotz robots, an internetconnected bunny-shaped ambient electronic device, were given to participants and their acceptance was tracked over seven months. Many participants lost interest after the initial excitement disappeared, but $10 \%$ of the subjects remained active until the end of the experiment.

In [3], a dinosaur robot (Pleo) was given to families for several months and it was studied how the experiences met with the high user expectations due to the price and the advertisements. In the reality, the robot skills were not enough sophisticated to satisfy the participants after the initial novelty effect faded away and Pleo was switched on rarely.

This study focuses on a domestic social robot, namely, the owners of Sony AIBO robots (Fig. 1) were analyzed. This product brand included quadruped autonomous entertainment robots which had a behavior-based architecture to exhibit a life-like impression. These robots walk around the room, interact with the owner and switch between probabilistic state machines to show rich behaviors and engage the owners. Several papers have been focused on AIBO in the past decade, but the heavy users were not examined. In [5], the on-line forums for Sony AIBO were analyzed to investigate the relationship between the robots and their owners. According to the posts on the internet, people developed an emotional connection to these robots, but they rarely attributed moral standing towards them.

The temporal change of an attitude can be examined with longitudinal studies where the same people are tracked over months $[2,3,4,7]$, but this is time-consuming with unexpected technological complications and scheduling user interviews. To the best knowledge of the authors, all past HRI researches organized short weekly or monthly sessions for the participants together with the robot $[2,3,4,10]$ before reaching the acceptance phase. The participants in this paper owned Sony AIBO for years and they still run these robot dogs time to time what is a fundamental difference from e.g. [3]. The earlier studies had also a challenge to recruit enough participants to allow statistical calculations for significant trends in the data, except [7].

The subjects in this experiment lived with commercial, social robots day by day for years. Since Sony AIBO was discontinued long ago when this survey was conducted, people were reachable who owned these robots for more 
than ten years. Furthermore, the Sony AIBO community on the internet was still active with new members who bought these social robots from secondhand sources. At the same time, newcomers and experienced owners could participate in this experiment, therefore, the subjects of this paper were beyond the acceptance phase and used their robots for years. The paper tries to answer the following research questions:

- How does the length of ownership affect the perception of the robot?

- Is there any significant difference between Westerners and Japanese people?

- Does the age (young/middle age/old) change the users' opinion?

- Does the gender make any difference?

\section{Questionnaire}

A questionnaire (Appendix) was conducted to get the opinion of people about their Sony AIBO robots. A flexible design was chosen with many Likert-type items which were easy to understand and fill out, but the participants also had the chance to give their own opinion in optional text fields. The expectation was that the target group (heavy users) had constant interactions with their robots, therefore, one question ensured that the participants run their social robot regularly. The other questions were related to the perception of their robots, how they feel about the existing software and which skills must be improved in AIBO. Eight questions asked basic information about the participants (gender, age, home location, profession) and the robot ownership (length, usage frequency, model preference). Question 9 investigated the impressions about the existing AIBO skills with 9-point Likert-type items (anchors: 1 - Strongly disagree, 3 - Disagree, 5 - Neutral, 7 - Agree, 9 - Strongly agree) and the following two concentrated on the expectations from a new software update. The adjectives of the Likert-type items for emotional perception were selected together with a psychologist who worked in the social robotics field and had experiences with questionnaires. Other Likert-type items queried technical aspects to identify wishes for specific skills of these social robots and the remaining questions collected answers about the connectivity options, autonomous behavior and possible user contributions. Several questions included text fields where the participants could enter additional comments in a free form, but those answers were analyzed in a previous paper of the authors [29].

78 fillings were collected from the members of an English speaking on-line AIBO forum (http://aibo-life.org) what is similar to 70 in [7] and 17 Japanese participants were reached via Facebook ad campaign, similar to [14]. Although Bartneck et al [1] distinguished the culture based on the nationalities, the current survey clusters the 61 forum members to Western culture and the 17 Japanese responses to Japanese culture. The authors made this decision to examine the stereotypical belief, similar to [1], that the Japanese people consider the robots with soul, unlike in the Western culture where the robots are recognized more as machines. Sony AIBO was a major hit in the Japanese market and the remaining user base was significant to form a single group in this survey for the culture variable.

The questionnaire was filled by 57 males and 19 females, since two participants did not reveal their gender, with a ratio $73 \% / 24 \%$, similar to the reported $69 \% / 31 \%$ gender ratio of AIBO owners in [28] and another on-line AIBO questionnaire with $64 \% / 36 \%$ in [1]. Although there was no question about the income and the wealth of the participants, the authors can explain this rate with the possible higher interest of the men in gadgets and they can afford more to buy expensive robots [16, 24]. The technical enthusiasm was also reflected in the professions because most owners were engineers, software developers or technicians $(27 \%$ for Tech in Fig. 2.b) and other occupations were between 1-15\% in Fig. 2.b.

Since this study focused on robot consumers with more years of ownership after technology acceptance phase, the analysis does not include such people who did not go beyond the technology adoption with Sony AIBO. Almost the half of these owners were young adults (under 40 years) or in middle age (40-60 years) and 5.13\% was old (Fig. 3.a). It is worth noting that $14.10 \%$ of the participant was under 25 years and their stories on the on-line forum given an insight of their intentions to buy these robots. Either they have got know AIBO in the recent years or they were children during the commercialization of AIBO and they could afford these robots after becoming an adult with income. The measured age distribution is very similar to the statistics of Japanese Sony AIBO owners reported in 2002 [28] what was an interesting similarity between the commercial and $2^{\text {nd }}$ hand periods in the product lifecycle. Fig. 3.b shows high retention rate for $20.51 \%$ of the participants who kept their robots for more than 10 years, $51.29 \%$ had AIBO between 2-10 years, but $28.21 \%$

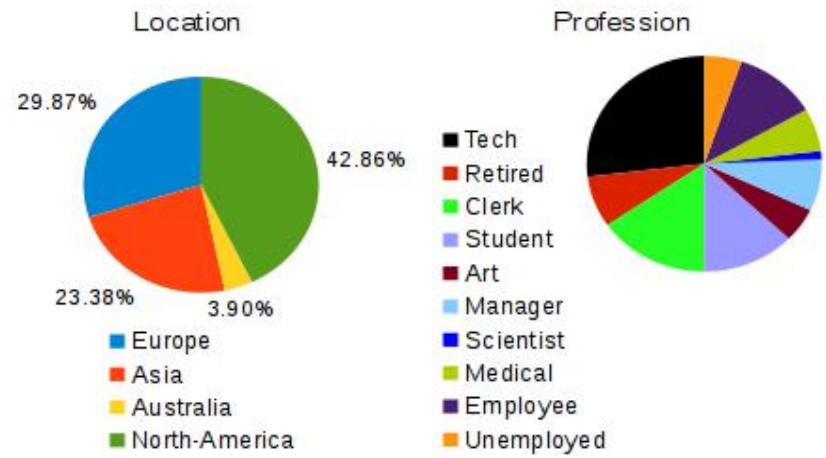

possessed AIBO for less than 2 years which is a high rate of newcomers.

Fig. 2 The home location (2.a) and profession (2.b) of the AIBO customers who responded to the questionnaire. 

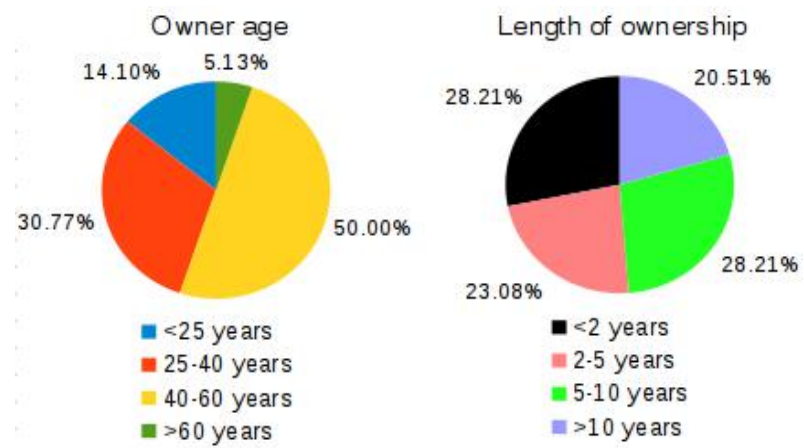

Fig. 3 The age (3.a) and length of ownership (3.b) of the participants

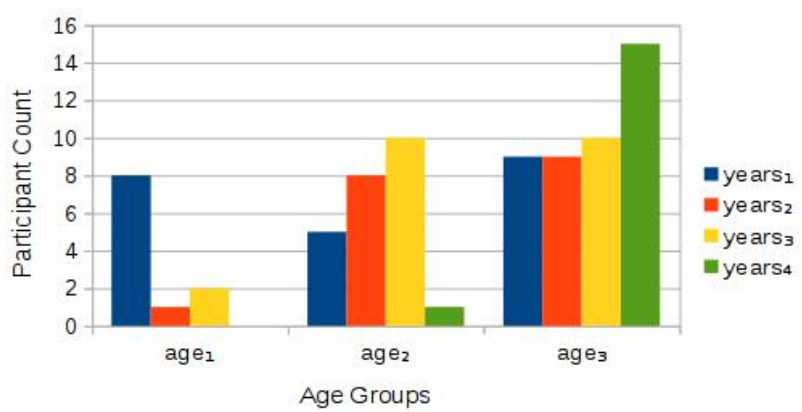

Fig. 4 Age distribution (age $e_{1}=<25$ years, age ${ }_{2}=25-40$ years, age $e_{3}=>40$ years) of the questionnaire participants in the function of the length of ownership (years ${ }_{1}=<2$ years, years ${ }_{2}=2-5$ years, years $3_{3}=5-10$ years, years $_{4}=>10$ years $)$.

Two questions in the survey asked about the age and the length of ownership. The first age group was under 25 years $\left(a e_{1}\right)$, the second between $25-40$ years $\left(a^{2} e_{2}\right)$ and the third over 40 years $\left(\right.$ age $\left._{3}\right)$. The length of ownership had four ranges: less than 2 years (years 1 ), 2-5 years (years 2 ), 510 years $\left(\right.$ years $\left._{3}\right)$ and over 10 years (years 4$)$. The majority of young generation (age $)_{1}$ possessed their robots for less than 2 years (years 1 ) on Fig. 4, the most typical duration

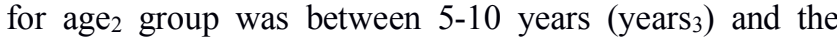
biggest portion of the oldest age group (age a owned their gadgets over 10 years (years 4 ). These results suggest that elder generations keep their robots longer and adults over 25 years (age ${ }_{2}$ and age $_{3}$ ) had their Sony AIBO for varying years (years 1 -years 4 ), the latter generations are newcomers and long-term customers at the same time.

\section{Overall Analysis}

Most questions in the survey were constructed with Likerttype items, they were grouped into subscales and their consistency was analyzed with Cronbach's $\alpha$ coefficients for sufficient trust in the overall reliability:

1. Emotional perception of the robot.

2. Emotional expectations from a new software.

3. Expected skill improvements in a software update.

\section{Connectivity options to the robot.}

The questionnaire did not have many responders (78) for an ideal quantitative data analysis, but the subscales had good $\alpha$ coefficients $(0.82,0.87,0.91,0.81)$.

\subsection{Exploratory Factor Analysis}

Exploratory factor analysis is a statistical method for uncovering the underlying structure of a questionnaire. All Likert-type items were investigated by this method to verify if the answers were coherent inside the subscales.

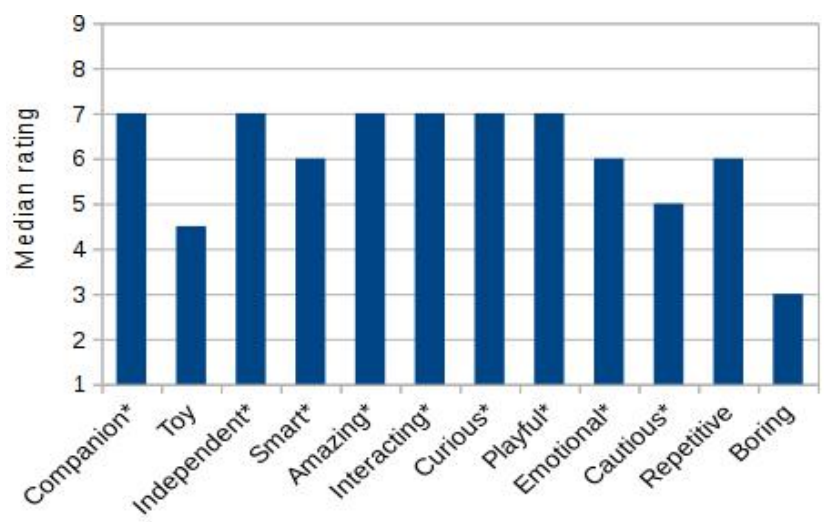

Fig. 5 Medin ratings of the existing robot software for Sony AIBO robots. The items in the second factor of the exploratory factor analysis are marked with an asterisk.

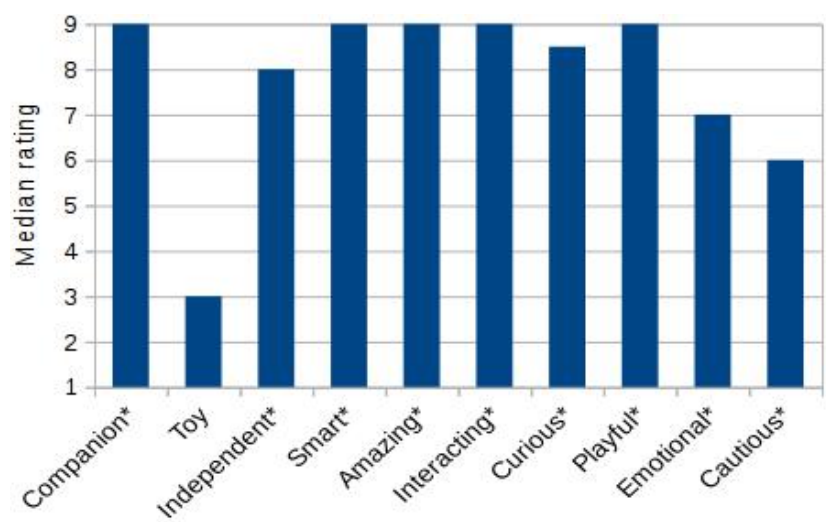

Fig. 6 Median ratings for wished features in a new software of Sony AIBO. The items in the fourth factor of the exploratory factor analysis are marked with an asterisk.

The Kaiser-Meyer-Olkin Measure of Sampling Adequacy was 1, the Bartlett's Test of Sphericity was significant under $\mathrm{p}<0.001$ for approximate of Chi-Square 3649.37 thus the measured variables were not normally distributed, but skewed. 12 items had eigenvalue over 1.00 and they expressed $78.17 \%$ of the total variance. A further evidence was for the coherence that several factors included most questions of certain subscales. Namely, the first subscale was found in the second factor (Fig. 5), the second subscale in the fourth factor (Fig. 6), the third 
subscale in the first factor (Fig. 7) and the fourth subscale in the third factor (Fig. 8).

The correlated items in the first factor were from the third subscale where AIBO owners wished to receive software updates to improve the robot intelligence (Fig. 7) except the toy skill. The second factor (Fig. 5) were from the first subscale and the owners perceived their robots with a positive attitude. They treated these machines as independent companions who were smart, amazing, interacting, curious, playful and emotional.

Some factors indicated relationship between items on a lower granularity:

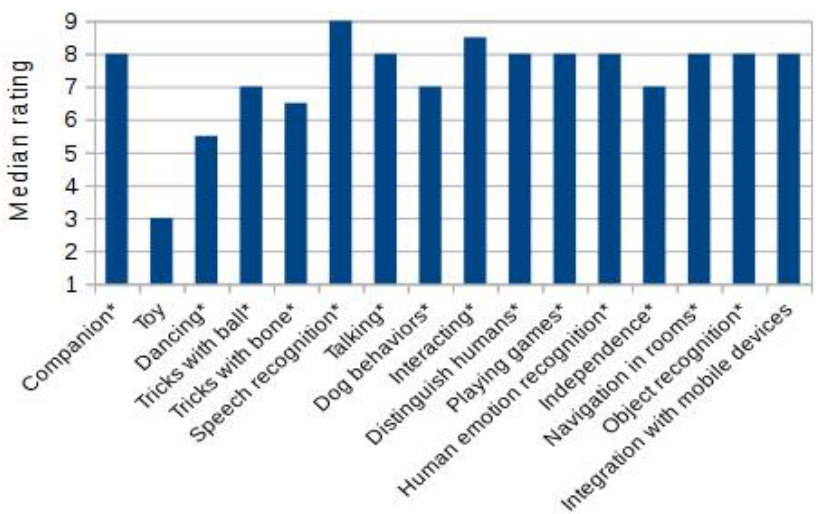

Fig. 7 Median ratings for improvements in the current robot software of Sony AIBO. The items in the first factor of the exploratory factor analysis are marked with an asterisk.

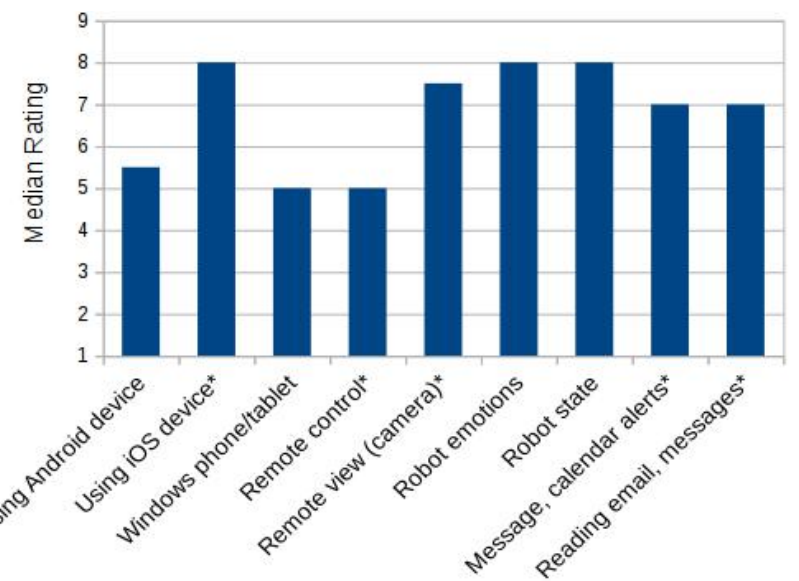

Fig. 8 Median ratings for wished connection options in a new software of Sony AIBO. The items in the third factor of the exploratory factor analysis are marked with an asterisk.

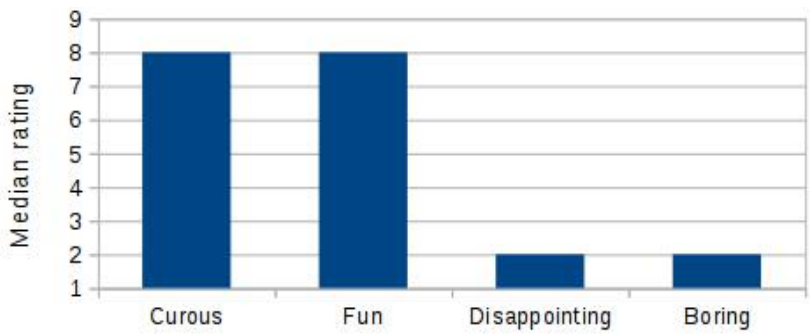

Fig. 9 Median ratings of the anticipated feelings caused by an autonomous personality in a forthcoming software for Sony AIBO.

- In the third factor, the Apple customers wished remote camera viewing, reading and alerts of messages and calendar events. On the other hand, Android and Windows Phone users preferred to control the robot remotely (fifth factor).

- People would not find a more autonomous software for the robot disappointing or boring according to the fourth factor.

- The sixth factor showed that the participants did not regard AIBO as a toy and they would not like to see improvements in features to make the robot a toy.

- Those owners would feel curious and fun a more autonomous mode in a new software who find the existing personalities boring or repetitive according to the seventh factor.

\subsection{Subscale Results}

Although Sony AIBO is an entertainment robot with limited capabilities, people attributed positive, life-like properties (e.g. companion, independent, smart) to the robot with 5-7 median ratings (Fig. 5) in the first subscale and it was not found boring (3) or a toy (4.5). On the other hand, the original software of AIBO was found quite repetitive (6) and the owners desired more sophisticated social skills from a new software with median ratings (7-9) of the second subscale on Fig. 6. Thus the owners judged these robots really "social" instead of a toy after years. This outcome was an opposite of an earlier experiment with Pleo in which the participants already treated the dinosaur robot a toy after some months [3].

The third subscale in the questionnaire focused on particular feature updates in the current software (Fig. 7). Enhancing the dances (5.5) and toy-like functions (3) had again lower interest. The tricks with plastic toys of the robot (ball, bone) and dog-like behaviors were not found so vital topics, most likely, because these features provide the entertainment aspect of the current software and the people are more eager to interact with the robots. The humanrobot interaction skills had the highest median ratings: companion (8), speech recognition (9), talking (8), interacting (8.5), distinguish humans (8), playing games (8) and emotion recognition (8). These skills shape a valuable emotional connection between the robot and people instead of watching repetitive entertainment behaviors. Worth to note that the participants would have liked to have enhanced autonomous features (navigation in rooms, object recognition) and further connection options following up the trend of portable handsets and tablets (integration with mobile devices) in the recent years. This result was aligned with Fig. 9 where the median ratings suggested that the robot customers had positive anticipations about a more autonomous software for Sony AIBO (Fig. 9). 
The connectivity options were queried in the fourth subscale (Fig. 8) and the majority was grouped in the third factor. The robots and Apple products are expensive, therefore, the higher classes can afford these items with a higher chance than people with lower income and they could be overrepresented in the survey responders although there were no question related to their wealth. The participants wished to connect their bots to iOS devices with the highest ranking (8) while Android had a moderate result (5.5), and according to the low market share, Windows devices had the lowest rating (5). Interestingly, people would like to see the robot state, emotions and camera image with median rankings $7.5-8$, but the remote control had a lower interest (5). This phenomenon can be originated that the people can associate a remote controlled robot to a soulless machine and making AIBO a toy was not an unattractive skill for the robot owners in Fig. 5, 6 and 7. Receiving alerts about SMS, calendar events or reading email was popular and scored to 7 .

To sum up this section, the exploratory factor analysis confirmed that the subscales were defined consistently and reliable results can be expected from the further examination. Looking at the median ratings of the Likerttype items, the participants had great desire to make the robot an autonomous companion which can interact with humans and connect the robot with the latest gadgets, but the repetitive behaviors for entertainment and acting as a toy were out of interest.

\section{Analysis of the Independent Variables}

The questionnaire responses were examined from gender, age, length of ownership and culture points of view to see how these independent variables affected the ratings. Each variable had a corresponding null hypothesis to be examined. If a variable had 2 categories, the Likert-type items were evaluated with Mann-Whitney test, for more than two categories, Kruskal-Wallis tests were performed. Depending on the results of these tests, the null hypotheses were either accepted or rejected.

Any difference in the median ratings of the Likert-type items were reported in the following subsections when they were higher than 1 and their $p$-values were less than .25 . In this way, the paper includes some insignificant results, but the authors wanted to keep these items because they fit in the tendencies of the significant items. Greater p-values were ignored.

In the last two subsections, the user contribution and the robot model preference were analyzed by the independent variables separately because these questions did not use Likert-type items.

\subsection{Gender}

Null hypothesis (H1): The male heavy users see a social robot as a machine and the female as a companion.
57 participants were male and 19 female from the survey respondents. The gender defined an independent variable with 2 categories and all Likert-type items were evaluated with Mann-Whitney test. The median ratings of women were all the time higher for those items where the robot was treated as a living being, the significant differences are shown on Fig. 10. Women attributed more human feelings to the existing software hence [1] Companion $(\mathrm{p}=.024)$, [1] Smart $(\mathrm{p}=.004)$, [1] Amazing $(\mathrm{p}=.021)$ and [1] Curious $(\mathrm{p}=.000)$ received more positive ratings by 2 . This attitude was also reflected in their wishes for improvements with [3] Human emotion recognition $(\mathrm{p}=.085)$.

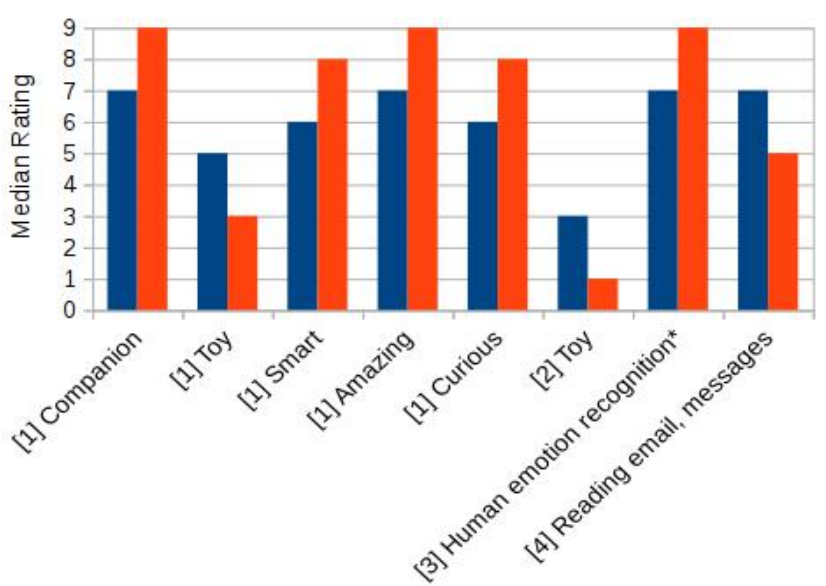

Fig. 10 Median ratings for items with considerable difference between male (blue) and female (red) answers whose number in square brackets refers to the subscale of each item. All components have significance under .05 except those which are marked with an asterisk.

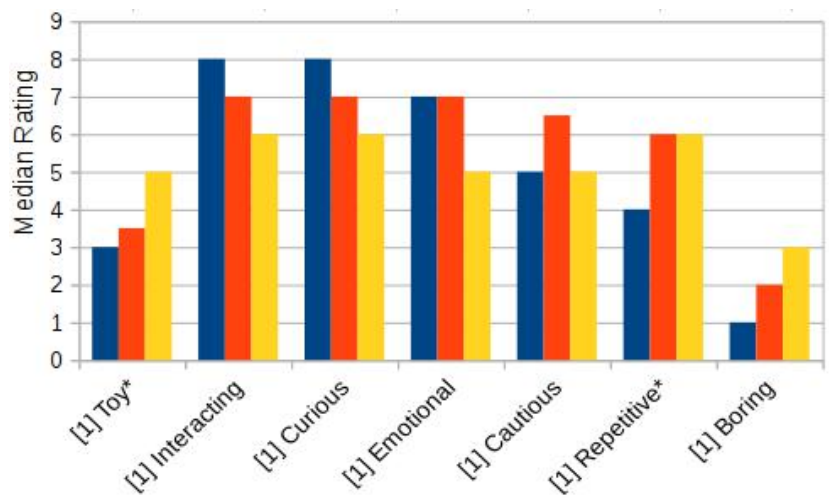

Fig. 11 Median ratings for items in the first subscale between age groups (blue for age $\mathrm{e}_{1}$, red for age $\mathrm{a}_{2}$ and yellow for $\mathrm{age}_{3}$ ). The number in square brackets refers to the subscale of each item. All components have significance under .05 except those which are marked with an asterisk. 


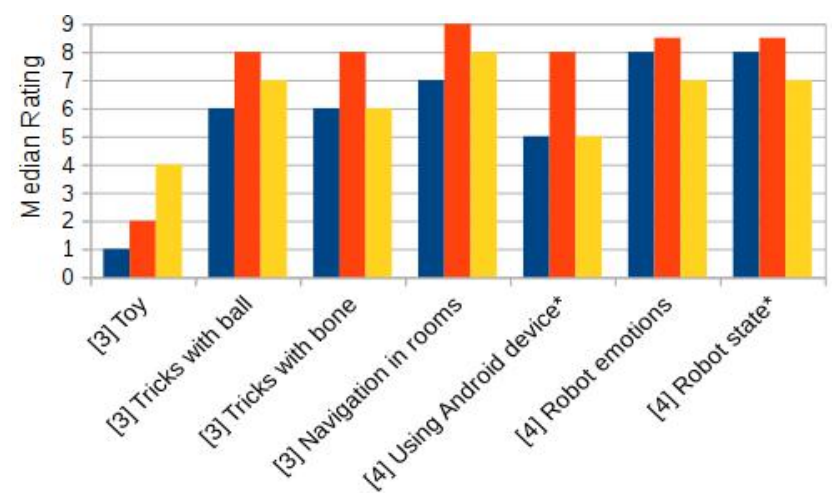

Fig. 12 Median ratings for items in the third and fourth subscales between age groups (blue for age $e_{1}$, red for age $\mathrm{e}_{2}$ and yellow for $\mathrm{age}_{3}$ ). The number in square brackets refers to the subscale of each item. All components have significance under .05 except those which are marked with an asterisk.

Males rated [1] Toy $(p=.004)$ and [2] Toy $(p=.004)$ with higher median by 2 , therefore, they regarded the robot to a greater extent as a machine. Similarly, men found more important to read e-mails and messages by the robot because they rated [4] Reading email, messages $(p=.031)$ higher by 2 .

As the common sense suggests, women tended to be more emotional in their ratings while the men were technology-minded. The H1 null hypothesis was accepted for the gender variable.

\subsection{Age}

Null hypothesis (H2): The younger heavy users are more technology-minded while the elder look the social robots as a companion.

11 participants were under 25 years $\left(a^{2} e_{1}\right), 24$ between 25-40 years $\left(\mathrm{age}_{2}\right)$ and 43 over 40 years $\left(\mathrm{age}_{3}\right)$. The age defined an independent variable with three categories and all Likert-type items were evaluated with Kruskal-Wallis test. Fig. 11 shows the items from the first subscale which had a significance value below or close to .050 and two main tendencies can be observed. On one hand, the older people the less positive they were about scoring the existing skills of the robot what can be seen for [1] Interacting $(p=.047),[1]$ Curious $(p=.027)$ and [1] Emotional $(\mathrm{p}=.000)$ on Fig. 11. On the other hand, the older age groups associated the robot with more negative properties by [1] Toy $(\mathrm{p}=.096)$, [1] Repetitive $(\mathrm{p}=.065)$ and [1] Boring $(\mathrm{p}=.008)$. The exceptional [1] Cautious $(\mathrm{p}=.011)$ age $_{2}$ found the robot more cautious than age $e_{1}$ and age ${ }_{3}$ groups.

There was no significant difference between the answers in the second subscale, but the third and fourth subscale revealed on Fig. 12 that the age 2 group tended to be more eager to see improvements in autonomous features of a new software with [3] Tricks with ball $(p=.015)$, [3] Tricks with bone $(\mathrm{p}=.013)$ and [3] Navigation in rooms $(\mathrm{p}=.013)$. Similar to this trend, age 2 was most interested in connectivity with Android phones and view [4] Robot emotions $(\mathrm{p}=.011)$ although [4] Using Android device $(\mathrm{p}=.059)$ and [4] Robot state $(\mathrm{p}=.113)$ were not significant. Older age groups recognized the robot rather as a toy (Fig. 11) and the same trend was measured for the wish of improved toy features with [3] Toy $(p=.027)$ in Fig. 12 . This may be originated in some reappearing traits from childhood in old age.

The results did not reflect the expectations of the null hypothesis (H2), elder people did not perceive these social robots as a companion to a greater extent and younger generations were not more eager about the technology side of these robots. Therefore, $\mathrm{H} 2$ was rejected.

\subsection{Culture}

Null hypothesis (H3): The Japanese heavy users do not rate their robots more positively than Westerners.

The cultural background of the participants was examined to compare Western people with Japanese. 61 responders were from Europe, North-America and Australia. Since these samples were collected from an English speaking AIBO forum and a closed Facebook group, the authors made sure that it did not contain any responses from migrated Japanese citizens in these countries. This original version of the questionnaire was in English.

17 Japanese fillings were gathered with a targeted Facebook ad campaign in Japan. The survey was localized to Japanese language and the filters of the campaign ensured that native Japanese people filled this variant. Additional evidences of the correct sampling were a special answering pattern to the occupation question by Japanese people and the common lake of answers to the free form entries.

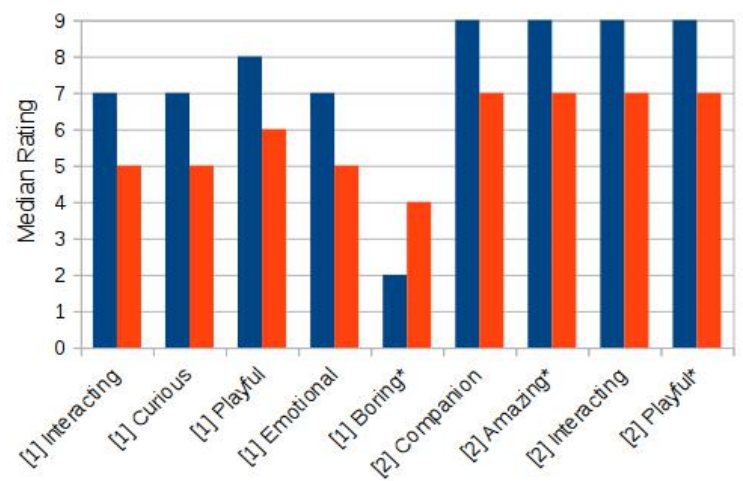

Fig. 13 Comparison of median ratings in the first and second subscale with different cultural backgrounds (blue for Westerners, red for Japanese). The number in square brackets refers to the subscale of each item. All components have significance under .05 except those which are marked with an asterisk. 


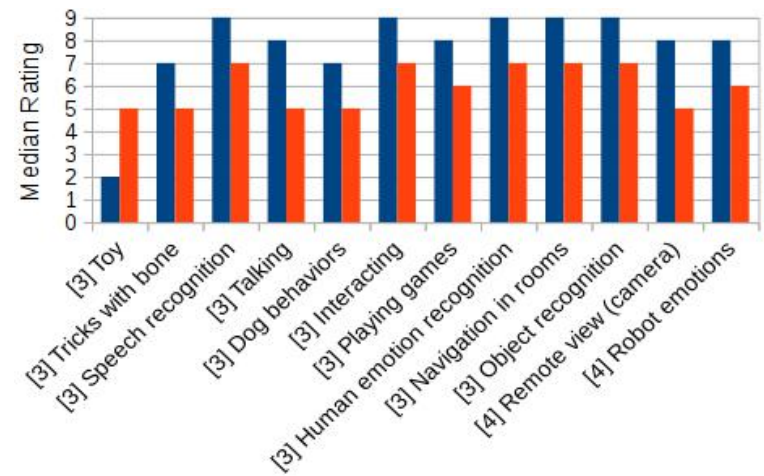

Fig. 14 Comparison of median ratings with different cultural backgrounds (blue for Westerners, red for Japanese). The number in square brackets refers to the subscale of each item. All components have significance under .05 except those which are marked with an asterisk.

The culture defined an independent variable with 2 categories, therefore, all Likert-type items were evaluated with Mann-Whitney test. The existing programs and wishes for new software were slightly less attractive for the Japanese in Fig. 13. The median ratings of [1] Interacting $(p=.001),[1]$ Curious $(p=.017)$, [1] Playful $(p=.006),[1]$ Emotional $(p=.008),[2]$ Companion $(p=.047),[2]$ Amazing $(p=.090),[2]$ Interacting $(p=.001)$ and [2] Playful $(p=0.150)$ followed tendencies of the Westerners, but they were lower by 2 . Fig. 14 has a similar pattern, the Japanese participants scored the feature improvements and connectivity options lower by 2-3 with [3] Tricks with bone $(p=.011)$, [3] Speech recognition $(p=.008)$, [3] Talking $(p=.006)$, [3] Dog behaviors $(p=.005)$, [3] Interacting $(p=.033)$, [3] Playing games $(\mathrm{p}=.017)$, [3] Human emotion recognition $(\mathrm{p}=.008),[3]$ Navigation in rooms $(\mathrm{p}=.047),[3]$ Object recognition $(p=.002)$, [4] Remote view $(p=.001)$ and [4] Robot emotions $(\mathrm{p}=.001)$. Furthermore, Japanese found the existing software quite [1] Boring $(\mathrm{p}=.081)$ in Fig. 13, but they wanted to see enhancements in [3] Toy $(p=.004)$ features in Fig. 14.

Since the Japanese people were more negative about Sony AIBO, the null hypothesis (H3) was accepted.

\subsection{Length of Ownership}

Null hypothesis (H4): The more years a heavy user owns a social robot without content updates the more robot acceptance decreases and he/she loses interest over time.

The questionnaire results were analyzed in the function of length of ownership with four categories. 22 participants have been owned the robots for less than 2 years (years 1 ) and 18 between $2-5$ years (years 2 ). These people were new members in the AIBO community years after the product discontinuation. 22 have been experienced these robots between 5-10 years (years 3 ) and 16 over 10 years (years 4 ). Because the independent variable had more than two categories, the Likert-type items were evaluated with Kruskal-Wallis test.
Fig. 15 shows nine items and almost all items had significance value over .050 . Despite the common sense suggests that the consumer interest must decline after years of usage without software updates, the results did not reflect this expectation. In particular, the existing software was evaluated in the first subscale and there were no significant decline in the anthropomorphic characterization. The owners with more than 10 years-long experience rated the robot with existing software more [1] Amazing ( $p=.137)$ and a [1] Companion ( $\mathrm{p}=.266)$ while they wished more [4] Remote control $(\mathrm{p}=190)$. After 5 years of ownership, the need for autonomous and social features were increased. Years 3 and years4 groups were wished a more [2] Curious $(p=.154)$ and [2] Playful $(p=.108)$ robot which is eager for [3] Talking $(\mathrm{p}=.165)$ and [3] Interacting $(\mathrm{p}=.011)$ with its owner. [3] Toy $(p=.160)$ feature was an odd-one-out feature because longer ownership decreased the desire for a toy robot although the years 4 group broke this tendency. In an earlier work [1], the ownership did not influence the attitude of the people towards robots, but Fig. 15 suggests that the owners will appreciate their robots more after 5 years. The null hypothesis (H4) was rejected because of these results.

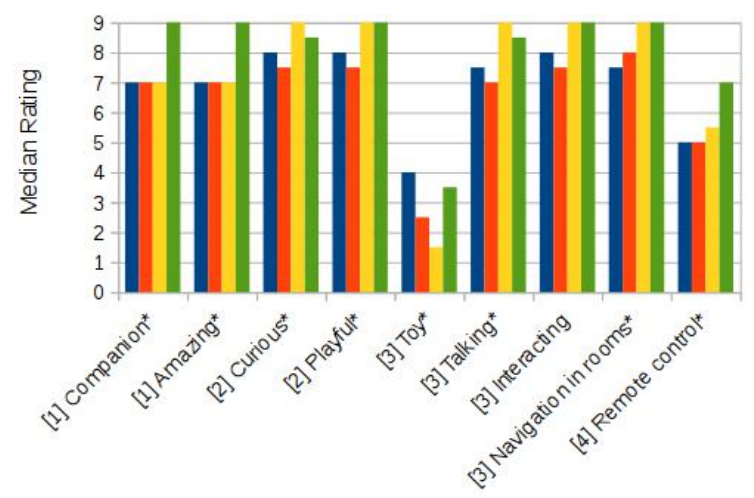

Fig. 15 Comparison of median ratings of participants with different length of ownerships (blue for years 1 , red for years 2 , yellow for years 3 and yellow for years 4 ). The number in square brackets refers to the subscale of each item. All components were marked with an asterisk because their significance were over .050.

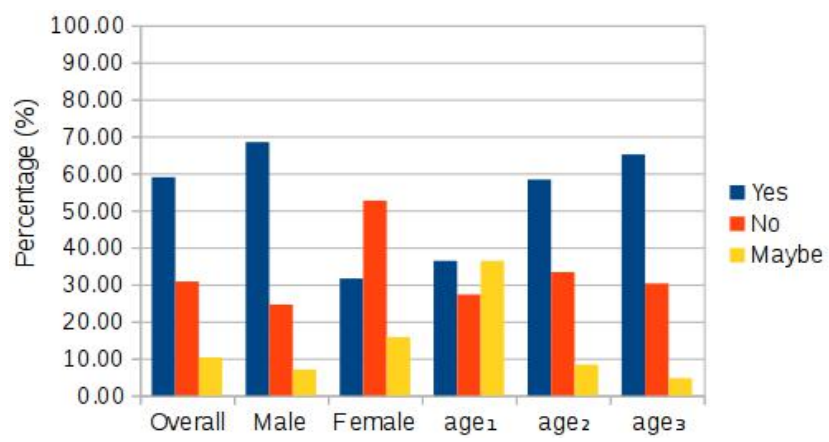

Fig. 16 Overall answers for user contribution question and details for gender and age (age a $_{1}=<25$ years, age $e_{2}=25-40$ years, age ${ }_{3}=>40$ years $)$ conditions. 
One question asked the owners if they would contribute new tricks for the robot software with a motion editor application. Fig. 16 shows that almost two-third of the users $(58.97 \%)$ expressed willingness to create new content for the robot, one-third $(30.77 \%)$ refused and $10.26 \%$ was unsure. As Section 4.1 confirmed, men were more focused on the technology side of the robot and males expressed double chance $(64.42 \%)$ for contribution compared to females $(31.58 \%)$ in Fig. 16 and women were twice as much unsure (males $7.02 \%$ vs. females: $15.79 \%$ ).

The traditional stereotype suggests that elder people are less open to the new technologies and younger generations catch them up more easily. Fig. 16 presents the opposite, the elder the owners are, the higher chance they will use their computer to create new motion content (age 1 : 36.36\% $<$ age $_{2}: 58.33 \%<$ age $_{3}: 65.12 \%$ ) and older generations were less hesitant about to make this decision (age : $36.36 \%>$ age $_{2}: 8.33 \%>$ age $_{3}: 4.65 \%$ ). Otherwise all age group rejected the contribution around $30 \%$.

When the answers were analyzed from the length of ownership condition, the people with over 10 years-long ownership (years 4 ) were the most eager $(87.50 \%)$ to create new motions for their robots, other owners given around $50 \%$ (years $1-3$ ). It is noteworthy that the newcomers (years 1 ) were the most hesitant $(22.73 \%)$ to make any content, the half of the owners with 2-10 years-long experience (years23) had no intention for contribution.

Japanese society is regarded acceptant for high-tech compared to other Western countries. This phenomenon is reflected in the culture condition on Fig. 17. $82.35 \%$ of the Japanese owners were keen to create new motions for the robot, $17.65 \%$ were not interested in and none of them were unsure.

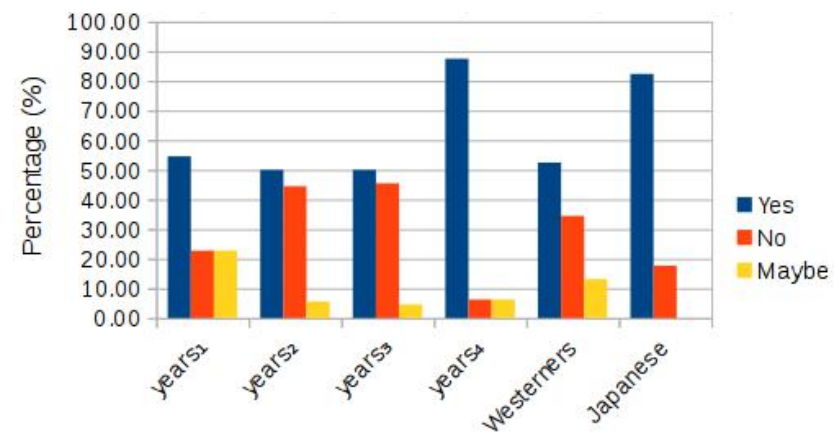

Fig. 17 Detailed answers for the user contribution question from length of ownership (years ${ }_{1}=<2$ years, years $2=2-5$ years, years ${ }_{3}=5-10$ years, years $_{4}=>10$ years $)$ and culture conditions points of view.

\subsection{AIBO Model Preference}

The Sony AIBO brand had several generations and every model had different advantages and disadvantages. The following list describes the most important characteristics of each product without going into technical details. These points were considered by the buyers when they decided to acquire a specific model:
- ERS-1xx: The first model in the series. More autonomous and advanced emotional personality was implemented in these robots. It had a charging station, but it could not execute the self-docking process. Despite the fragile body or repetitive behaviors many owners liked ERS-1xx because of the high degree of freedom, intensive interactions in the growth stages of the personality and the impulsive exploration mode. Secondhand price: $\$ 300-400$.

- ERS-2xx: The emotional model was not so complex in this successor. Many software were available for ERS-2xx, but each provided different features (e.g. self-docking, autonomous exploration). The owner had to change the memory card to switch between the personalities. The appearance of these models were much more clean compared to ERS-1xx. Secondhand price: $\$ 500-700$.

- ERS-3xx: This model was a simplified version of ERS-2xx with a lower price tag and the cute design to target female customers [28]. It had no wireless connectivity and less software were shipped for ERS$3 x x$. Secondhand price: $\$ 400-500$.

- ERS-7: The latest model had the best hardware and all previous skills of ERS-2xx were combined into a single firmware (Mind software). The robot docked itself to the charging station, did tricks, communicated with the owner, played with its toys and explored the surroundings although the latter skill was not so adventurous as in ERS-1xx. Secondhand price: $\$ 1800-2200$.

The most advanced model was the Sony ERS-7 which still maintains high price on the secondhand market. The ERS-2xx provided the same features scattered in various software and the lower price range (\$500-700) balance this disadvantage. In general, ERS-7 and ERS-2xx were the favorite models in Fig. 18 and 19 and ERS-7 was the top rated for being the most advanced. Third place went for the ERS-1xx whose interaction skills and autonomous mode compensated the weak hardware design and the lack of features. The ERS-3xx models were rated worst caused by the missing connectivity and software options. When the user preferences were reviewed for the gender condition, the males chosen a favorite model less frequently than females and the women preferred ERS-2x0 in the first place over ERS-7 exceptionally.

The age condition revealed in Fig. 18 that young people $\left(\right.$ age $\left._{1}\right)$ had no clear preference for any Sony AIBO, they were interested in all models. The ERS-2xx and ERS-7 series were in tie for $<10$ years in the length of ownership condition (Fig. 19), but the latest model was a winner for years 3 group. The Japanese people tend to select only one favorite which can be originated in the emotional attachment after buying their first model. The model preference of Westerners followed the overall results in Fig. 18 while Japanese rated all models to $35.29 \%$ except the ERS-3xx to $17.65 \%$. 


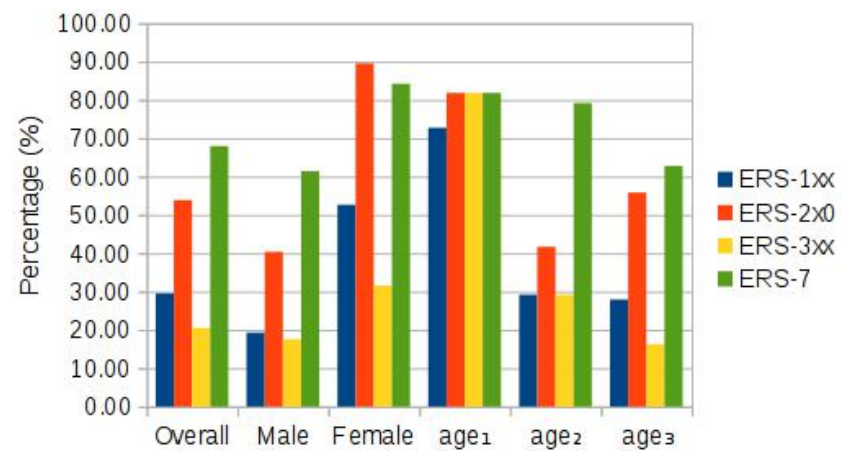

Fig. 18 Preferred AIBO models. One participant could select multiple favorite models. This diagram contains the overall results and the details for gender and age $\left(\right.$ age $_{1}=<25$ years, age $2=25-40$ years, age $_{3}=>40$ years) conditions.

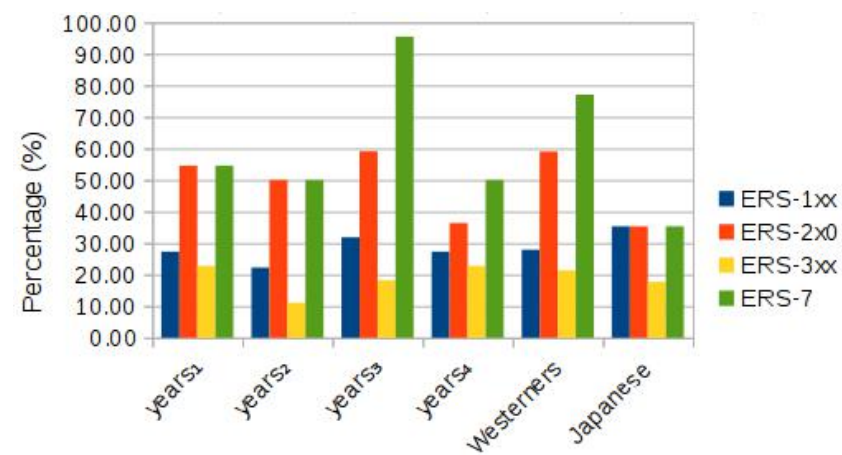

Fig. 19 Preferred AIBO models. One participant could select multiple favorite models. This diagram contains detailed answers from length of ownership (years ${ }_{1}=<2$ years, years ${ }_{2}=2-5$ years, years ${ }_{3}=5-10$ years, years $_{4}=>10$ years $)$ and culture conditions points of view.

\section{Discussion}

\subsection{Gender}

The H1 null hypothesis was constructed with considering the differences between male and female social behavior and their social roles in society [27] and it was accepted for the gender variable because the female participants were more emotional in their ratings while the men were more technology-minded. The past researches $[15,17,20]$ had mixed results within the Japanese society. A survey of female and male Japanese at an exhibition did not find a variance in their opinions about robots after an interaction [15] and an online survey among Japanese people had the same result in [20]. However, Japanese students were asked about the attitudes towards robots without actual interaction in [17] and the female students were more positive about the emotional interactions with robots than male, similar to H1. Most likely, there is no gender difference in the Japanese society according to [15] and [20], but some subgroups (younger generations?) can show some variation. On the other hand, female Italians attributed more positive feelings for robots than males in a questionnaire [26] what strengthens $\mathrm{H} 1$ since the majority of the participants in this paper were from Western societies. The results in this regard were more close to cultural expectation according to the literature, similar to the next subsection.

\subsection{Age}

H2 was rejected because elder people did not perceive Sony AIBO as a companion and younger generations were not more interested in the technology side of these robots. This result is similar to [24] in which Australian participants over 50 years disliked the robotic pets, younger Italian generations had more positive feelings towards robots in [26] and Ezer reached the same conclusion in [25] with American citizens, the acceptance was higher among younger adults in pre-adoption phase (without firsthand experience with robots). However, two studies $[15,20]$ found the opposite in the Japanese society. Younger Japanese generations liked the robots less than elder people, but this discrepancy can be explained with the different sampling. $[15,20]$ were interviewed only Japanese while this paper included mainly Westerners. The revealed attitudes here and in the literature strongly suggest that the generations in the Japanese and Western cultures have the opposite preferences. However, the limited sample size was not enough to carry out more analysis about this assumption within this work.

\subsection{Culture}

The common stereotype suggested in the past that the Japanese people love robots more than Westerners. Some early attempts tried to understand and explain this belief by comparing the cultures [22] instead of executing experiments with humans. Later surveys [1, 18] revealed the opposite, Japanese are not so positive towards robots and they are worried about the social and emotional impacts of robots in their society. The null hypothesis (H3) in this paper was constructed in accordance to these discoveries and the results confirmed $\mathrm{H} 3$, therefore, it was accepted. Worth to note that Japanese were more negative than Westerners in all cases what strengthens the results in [19] where Australian people were more positive about likeability and intelligence of a humanoid robot. Other works $[1,18]$ showed no difference, but the results in this paper and [19] require further analysis to clarify the roots of the unexpected antagonism of the Japanese people towards robots.

\subsection{Length of Ownership}

The rejection of $\mathrm{H} 4$ was a positive finding. The common sense suggests that people leave things behind after their utility value decreases over time, but the heavy users of Sony AIBO acted in the opposite way after 5 years of the ownership. 
Getting people into acceptance phase is challenging because the high price tag of the robots induces high expectations from the users. Some participants did not finish a six months-long experiment with Roomba vacuuming robots [23] as they required too much maintenance. Though Graaf et al [7] analyzed the robot owners on a different timescale (half year vs. multiple years here) and $5-10 \%$ of their participants showed constant interest for Karotz robot in a longer period. Other people did not become a power user because Karotz robot did not offer more functions than a modern smart phone and they did not feel the robot useful, similar to other past works $[3,11]$.

The authors believe that the acceptance for Sony AIBO robots did not decrease over the years in the current study because their software were designed to develop emotional attachment with their owners and their main functions were not intended to replace a computer.

\subsection{User Contribution}

The user contributions strengthened the results of Section 4.1 and older generations were more likely to contribute, especially after owning a robot for more than 10 years. It was also revealed that the Japanese participants were more eager and less hesitant to make technical contributions unlike Westerners. This finding is exciting compared to the long decision making in Japanese companies or the hesitation of Japanese people to say black-and-white decisions straightforward [21]. Maybe the anonymous questionnaire on the web allowed the Japanese participants to leave their comfort zone and they could really express what they think. The higher willingness for contribution of Japanese people was a bit surprising when this result was compared to the negative trends of Japanese answers for the culture variable (Section 4.3).

\subsection{AIBO Model Preference}

ERS-3xx had the worst score in almost all cases in Fig. 18 and 19 which suggests that people would like to use a social robot with rich skills and connectivity options. The low price tag cannot compensate the missing capabilities if similar robots with more advanced intelligence are on the market. Despite a Sony ERS-7 costs 3-4 times more than other series, people tend to prefer this model as a result of the most developed hardware and software.

\subsection{Social Robot Design}

Two papers proposed guidelines to improve the design of social robots for long-term human-robot interactions. Leite et al. [11] presented a good review of this problem with a detailed discussion by accumulating the experiences of different robots in the research literature while [8] expressed their recommendations on a higher level. The authors reviewed the free-form answers of the questionnaire in an earlier work [29] and some proposals were already given to the literature. Based upon the quantitative analysis in this paper, additional suggestions are presented here in descending priority to complement the past works $[8,11]$ :

- The long-term ownership does not bias the tendencies of robot acceptance what is expected in a certain cultural background and life stage. However, the literature review suggests that the acceptance must be examined with culture and age variables with sufficient sampling at the same time because the people's preferences vary in these dimensions significantly and they make hard to draw general conclusions.

- The age distribution of the owners remains stable, even long after the sales is stopped and the robot is traded on the secondhand market (see Section 2).

- The long-term ownership does not degrade the appreciation towards the robot after several years (see Section 5.3), but the owners desire the integration of the latest technologies.

- The robot should not replace the functions of a smart phone or a computer, especially with more hassle. The robot needs to differentiate itself from other machines with unique skills (see Section 5.3).

- Do not sacrifice essential skills to reduce the hardware costs otherwise consumers will not like the robot (see Section 5.6).

- The robot must adapt its personality with subtle differences according to the human gender, age and culture (Section 5.1, 5.2 and 5.4).

- Older people tend to treat the robot as a toy and they are less positive about the social skills, but they are more enthusiastic to create new content for the robot (Fig. 11, 12 and 16).

\subsection{Limitations}

Despite of the participants were recruited on a special internet forum and Facebook on the internet, only 78 active Sony AIBO owners were reached, but the authors believe that sample size was reasonable compared to 230 in [1] and 41 in [18] considering that conducting our survey was long after the discontinuation of Sony AIBO. The sampling was not representative for the general public, but the participants could provide a good indication about the typical users of entertainment robots and even beyond this group since Bartneck et al also found in [1] that owning a Sony AIBO did not result significantly different scores on their NARS questionnaire.

Since these robots were commercial, this study was essential to analyze the heavy users of an expensive robot from the market. The robots in past experiments were given to participants on a voluntary basis for free $[3,8]$. 
The sample size was moderate, the cultural variable could not be evaluated on a nationality level, and therefore, this category was reduced to a comparison between Westerners and Japanese people. Unfortunately, the results seemed to be biased by the Western majority for the gender and age variables (Section 5.1 and 5.2) when the results were compared to the literature. The authors still believe that the previous sections presented significant results and further exploratory studies are required with other social robots to confirm or complement the findings.

\subsection{Conclusions}

The heavy users of Sony robot dogs were studied in this paper after 10 years of the product discontinuation. Since these people owned their robots for years after the initial "wow" moment faded out, they were already in the robot acceptance phase.

The Westerner members of an active on-line community and Japanese owners via Facebook were reached to fill a questionnaire. The questions in this survey asked about the perception of their social robots and the desired improvements in the software. The internal consistency of the answers were verified with Cronbach's $\alpha$ coefficients for four subscales and the exploratory factor analysis mapped the questions into the predefined subscales correctly along with other findings. In overall, the participants rated their robots quite positively after so many years although they wished for many improvements in various functions. The answers were analyzed by four independent variables (gender, age, length of ownership, culture) and user contribution. Each independent variable had a null hypothesis and the Likert-type ratings were verified by Mann-Withney or Kruskal-Wallis tests depending on their categories. In gender case, the gender hypothesis (H1) was accepted, the male heavy users tended to have a technological perception of their robots while the female seen a companion in them. The hypothesis (H2) for age groups was rejected, the younger generations were not so tech-savvy and the pensioners did not recognize eagerly these social robots as a companion. The third hypothesis (H3) for culture variable was accepted, and the result was similar to [1], but the Japanese people were more negative about their Sony AIBOs what contradicts the common stereotype of the robot-loving Japanese society. The length-of-ownership hypothesis (H4) was unexpectedly rejected in a positive manner because the heavy users with over 5 years of ownership rated their bots significantly better. The user contribution question revealed that most heavy users are prepared to make new content expect the young and female. These results were turned into recommendations for social robot design and the limitations of the questionnaire were discussed.

The future work can include similar analysis with heavy users of other social robots which are developed to be a companion for people. The straightforward choice can be the future heavy users of the new Sony ERS-1000 model what revived the AIBO line back to the market in 2018. An interesting question how may alter the questionnaire findings if Sony will shift the emphasis in the new robot software from entertainment to companionship. However, executing our survey with the heavy users of other nonanimal (e.g. humanoid) robots can be also valuable because their appearances drive different expectations on the uncanny valley and this condition can alter the results. On the other hand, the authors believe that a comparison with a STEM or service robot would not be beneficial since the emotional attachment is essential towards social robots. If this connection is missing, it is unlikely that the owner will use the robot for many years without treating it other than a soulless machine.

\section{Acknowledgements}

The authors want to say thanks to all enthusiastic members of the aibo-life.org forums to fill out my questionnaire. Special thanks to Christoph Bartneck who shared his valuable research data to draw better conclusions in this paper.

\section{Compliance with Ethical Standards}

The authors declare that they have no conflict of interest.

\section{References}

[1] Bartneck C, Suzuki T, Kanda T, Nomura T (2007) The influence of people's culture and prior experiences with AIBO on their attitudes towards robots. AI \& Society, 21(1-2): 217-230

[2] Coninx A, et al (2016) Towards long-term social childrobot interaction: using multi-activity switching to engage young users. Journal of Human-Robot Interaction, 5(1): 32-67

[3] Fernaeus Y, Håkansson M, Jacobsson M, Ljungblad S (2010) How do you play with a robotic toy animal?: A long-term study of pleo. In: Proceedings of 9th international conference on interaction design and children ACM, New York, pp 39-48

[4] François D, Powell S, Dautenhahn K (2009) A longterm study of children with autism playing with a robotic pet: taking inspirations from non-directive play therapy to encourage children's proactivity and initiative-taking. Interact Stud 10(3): 324-373

[5] Friedman B, Kahn P H, Hagman J (2003) "Hardware companions?": What online AIBO discussion forums reveal about the human-robotic relationship. CHI Letters, 5(1):273-280

[6] Gockley R, Bruce A, Forlizzi J, Michalowski M, Mundell A, Rosenthal S, Sellner B, Simmons R, Snipes K, Schultz A, Wang J (2005) Designing robots for longterm social interaction. In: 2005 IEEE/RSJ international conference on intelligent robots and systems (IROS), pp $1338-1343$

[7] Graaf, MM, Ben Allouch S, Dijk JA (2014) Long-term evaluation of a social robot in real homes. 3rd Intl Symposium on New Frontiers in Human-Robot Interaction (AISB)

[8] Graaf MM, Ben Allouch S, Dijk JA (2016) Long-term acceptance of social robots in domestic environments: Insights from a user's perspective. AAAI 
[9] Kertész C and Turunen M (2015) Community driven artificial intelligence development for robotics. In: 12th Int Conf on Informatics in Control, Automation and Robotics (ICINCO), Doctoral Consortium and Poster.

[10] Koay K, Syrdal D, Walters M, Dautenhahn K (2007) Living with robots: investigating the habituation effect in participants' preferences during a longitudinal human-robot interaction study. In: The 16th IEEE international symposium on robot and human interactive communication (RO-MAN), pp 564-569

[11] Leite I, Martinho C, Paiva A (2013) Social robots for long-term interaction: a survey. Int $\mathrm{J}$ Soc Robot 5(2):291-308

[12] Mori M (1970) The uncanny valley. Energy 7(4):3335

[13] Salter T, Dautenhahn K, Bockhorst R (2004) Robots moving out of the laboratory-detecting interaction levels and human contact in noisy school environments. In: Proc. of 13th IEEE international workshop on robot and human interactive communication (ROMAN), pp 563-568

[14] Samuels D and Zucco C (2012) Using Facebook as a subject recruitment tool for survey-experimental research. Working paper, Social Science Research Network.

[15] Nomura T et al (2005) Questionnaire-based research on opinions of visitors for communication robots at an exhibition in japan. In: Proc. of IFIP Conference on Human-Computer Interaction, pp 685-698

[16] Yoldas S (2012) A Research About Buying Behaviours of Online Customers. MSc Thesis, University of Roehampton.

[17] Nomura T, Suzuki T, Kanda T, Kato, K (2006) Altered attitudes of people toward robots: Investigation through the Negative Attitudes toward Robots Scale. In: Proc. AAAI-06 Workshop on Human Implications of Human-Robot Interaction, pp 29-35

[18] Haring KS, Mougenot C, Fuminori ONO, Watanabe K (2014) Cultural differences in perception and attitude towards robots. International Journal of Affective Engineering 13(3):149-157

[19] Haring KS, Silvera-Tawil D, Takahashi T, Velonaki M, Watanabe K (2015) Perception of a humanoid robot: A cross-cultural comparison. In: Proc. of 24th IEEE international workshop on robot and human interactive communication (ROMAN), pp 821-826

[20] Nomura T, Sugimoto K, Syrdal DS and Dautenhahn K (2012) Social acceptance of humanoid robots in Japan: A survey for development of the frankenstein syndorome questionnaire. In: Proc. of 12th IEEE-RAS International Conference on Humanoid Robots, pp 242247

[21] Bernhauerova, M (2013) American vs. Japanese Management Style: Which One Yields Success. MG 201, Introduction to Functions of Management.

[22] Kaplan, F (2004) Who is afraid of the humanoid? Investigating cultural differences in the acceptance of robots. International Journal of Humanoid Robotics, 1: $465-480$
[23] Sung, J, Grinter RE, Christensen HI (2010) Domestic robot ecology. International Journal of Social Robotics. 2(4):417-29

[24] Zhan K, Zukerman I, Moshtaghi M, Rees G (2016) Eliciting Users' Attitudes toward Smart Devices. In: Proc. of the Conference on User Modeling Adaptation and Personalization, pp. 175-184

[25] Ezer, N (2008) Is a robot an appliance, teammate, or friend? Age-related differences in expectations of and attitudes towards personal home-based robots. Georgia Institute of Technology, $\mathrm{PhD}$ Dissertation.

[26] Scopelliti M, Giuliani M V, Fornara F. (2005) Robots in a domestic setting: a psychological approach. Journal of Universal Access in the Information Society. 4(2): $146-155$

[27] Norman D A (2004) Emotional Design: why we love (or hate) everyday things. New York, Basic Books.

[28] Fujita, M (2004) On activating human communications with pet-type robot AIBO. Proceedings of the IEEE, 92(11): 1804-1813

[29] Kertész C, and Turunen M (2017) What Can We Learn from the Long-Term Users of a Social Robot? In: Proc. Of 9th International Conference on Social Robotics (ICSR), 2017. 